\title{
Modular Workpiece Carrier System for Micro Production
}

\author{
Tobias Iseringhausen, Raphael Adamietz, Dirk Schlenker, and Alexander Verl
}

Fraunhofer Institute for Manufacturing Engineering and Automation IPA, Stuttgart, DE

\begin{abstract}
In micro production high lot sizes, which are required for costeffectiveness, are often not achieved, e.g. due to a high number of product variants or limited demand. Therefore new approaches for production like changeable manufacturing systems are demanded. In this paper a concept for a modular workpiece carrier system including a platform for sensors and actors is presented. The modular design allows the easy adaption of the manufacturing system to new products by the workpiece carrier system in terms of hardware and even the integration of additional process functionality.
\end{abstract}

Keywords: changeability, micro production, precision assembly, workpiece carrier.

\section{$1 \quad$ Introduction}

While product life cycles get shorter, production volumes vary and the number of product variants increases [1]. In some branches, especially the manufacturing of hybrid micro systems like sensors and other electro mechanical devices, nowadays often only low to medium lot sizes are reached, due to limited demand. As the conventional automated production with special purpose machines requires high lot sizes to be profitable, new approaches for efficient production are required [2]. Another aspect beside the lot sizes is the limited capability of manufacturing systems regarding additional functionality. For example, the integration of additional actors and sensors requires a large effort in terms of time and money, especially for the control adaption of the often closed systems, and causes related downtimes.

To cope with the changing requirements that the market imposes, increasingly changeable systems are required [3]. Further functionality is often provided by additional processes in the form of process modules. However, an increase of changeability can also be achieved by other system components. Generally, by improving system properties (changeability enablers) towards modularity, compatibility, mobility, universality and scalability, the changeability of the overall system is improved [3]. This applies to all levels of the factory, including the components level. One component, which is strongly linked to both the product and the production system, is the workpiece carrier. An increase of changeability of the workpiece carrier would improve the overall system changeability and offer a further possibility to adapt to product and process requirements. Furthermore, process functionality could be added to the 
workpiece carrier, which could enable further possibilities of system evolution on many levels.

\section{Related Work}

To increase changeability and enhance process functionality on component level by workpiece carriers, corresponding systems have been developed. For example, to manufacture 3D-MID devices in conventional PCB manufacturing lines a multi axis workpiece carrier has been developed [4]. For applications in milling machines an automated, reconfigurable fixture has been developed, which allows automated fixture planning and automated fixture configuration. It is equipped with sensors for control. Exchangeable jaws enable the reconfiguration [5]. To suppress self-induced vibrations during cutting on machine tools and thus increase surface quality and reduce shape deviations, active workpiece carriers with mostly piezoelectric actuators are a current research topic $[6,7]$. Due to the purpose of workpiece carriers, it is a wide-ranging topic with systems often adapted to very special purposes.

\section{Modular Workpiece Carrier System}

In this paper a concept of a workpiece carrier system designed for the application in micro production processes is presented. There is a focus on micro assembly, and therefrom requirements are derived. Micro assembly systems and micro assembly tasks are distinguished by some typical boundary conditions compared to conventional macro assembly. To determine these boundary conditions, a survey targeting microsystem manufacturers in Germany has been performed [8].

The most obvious difference between micro and macro is the size of microtechnical products. $87 \%$ of microtechnical devices fit into a volume of $100 \mathrm{x} 100 \mathrm{x}$ $100 \mathrm{~mm}^{3}$, while $30 \%$ are even smaller than $10 \times 10 \times 10 \mathrm{~mm}^{3}$ [8]. Small structures and functional elements demand positioning accuracies between 5 - 25 microns or even higher during assembly [8]. This imposes high requirements on the overall assembly system, like stiffness and precision, and the positioning technique itself. Furthermore, increased demands on cleanliness and defined, constant temperatures have to be regarded. Another characteristic is the high number of product variants. $75 \%$ of microtechnical products have up to ten or more variants [8].

The tracking of quality relevant parameters gains increasing importance. This applies particularly to many medical products, but the tracking requirements also exist in other industries (automotive, aerospace, etc.). Furthermore for high quality products, the data obtained by extensive tracking and tracing can be used for production optimization.

To comply with these requirements and increase the changeability and process functionality as described above, the concept of the workpiece carrier system is modularly designed to allow the adaption of the workpiece carrier by the easy exchange of functional elements like sensors and actors, so called smartFeatures. It consists of a modular electronic platform which is matched with a modular mechanical design. 
This allows a broad range of applications in micro production, nevertheless by the scalability of the system and operating distances even large scale systems are possible.

\subsection{Modular Electronic Platform}

The main items of the modular electronic platform are sketched in figure 1 below. The central component is a control unit, which is integrated into the mobile workpiece carrier. It allows data acquisition, processing and output of the connected sensors and actors. The communication within the workpiece carrier is done wireless if beneficial or tethered. The communication to the superior system, e. g. the machine control, is done wireless via a machine coupler, which is equipped with machine adapted control interfaces.

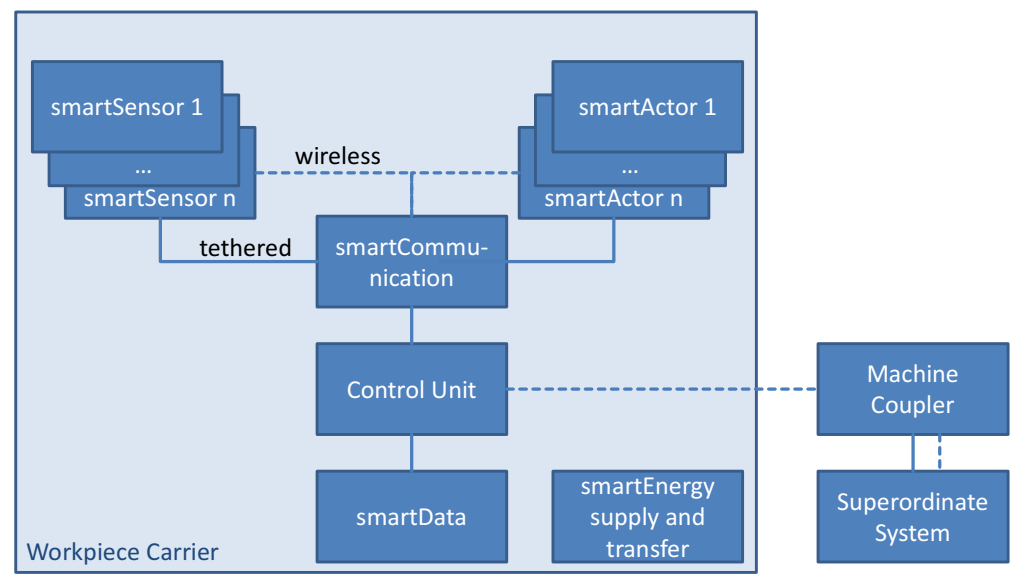

Fig. 1. Modular electronic platform

For energy supply a battery system and an energy transfer system with capability of wireless power is provided. Beside the control unit and machine coupler the further components are designed as modular. Thus it is possible to adapt the system to the requirements and conditions of the particular use case. For instance energy-saving smartFeatures combined with a high-capacity energy supply can be used for longterm applications with reduced processing power requirements. The smartSensors are capable of data acquisition and preprocessing to, for instance, reduce data volume within the electronic platform or increase accuracy by preprocessing close to the sensor to avoid signal interferences. Different types are drafted: one type with capability of high frequency applications, one low power type and one type designed for high accuracy. 


\subsection{Modular Mechanical Design}

The associated mechanical design of the workpiece carrier system is consistently modularly designed, see figure 2 below. The central element of the mechanical design is a workpiece carrier with frame architecture, see top element in the figure above. To facilitate the dissemination of the system, the outer dimensions and the outer mechanical interfaces refer to the standard DIN 32561. This standard describes dimensions and tolerances of a tray in the field of production equipment for microsystems. The mandatory elements of the electronic platform, control unit, communication as well as energy supply and transfer, are placed into the frame. Thus an encapsulation of the electronic components in rough environments like ultra-precision machining is ensured. The aperture within that frame architecture can now be individually designed with modules, considering the mechanical and electronic interfaces to the workpiece carrier and is matched to the size of $100 \times 100 \mathrm{~mm}^{2}$, which most microtechnical devices fit into, pursuant to [8]. For the integration of multiple modules distribution modules can be applied.
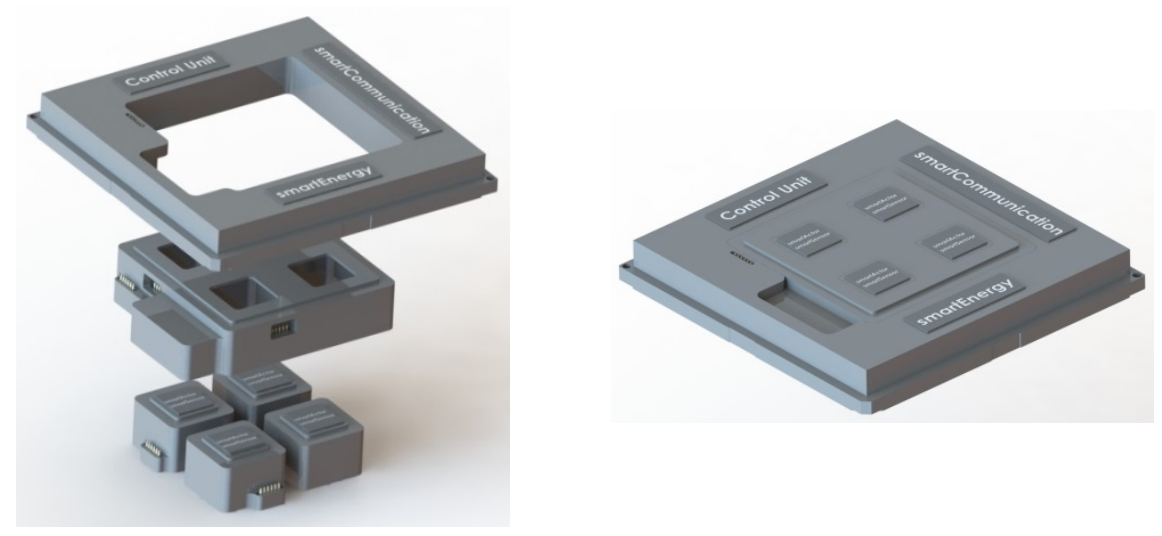

Fig. 2. Mechanical design of the workpiece carrier system

By flexible control interfaces of the machine coupler and even simple ones, like digital inputs and outputs, the system can be integrated into almost every machine with minor effort. The setup and programming of the workpiece carrier can take place outside the machines in specially designed set-up stations if required. Due to the compact design with low height and different sizes the mechanical integration is feasible in many, even limited, working spaces.

To give an illustrative draft of a workpiece carrier system, one example adapted for precision machining is sketched in the figures below.

Here modules with motor-driven clamping are inserted into the workpiece carrier. Additionally there is a module with inertial measurement unit and temperature sensor. It allows the measurement of the environmental temperature close to the workpiece and the detection of shocks during the manufacturing of sensitive parts. In the event of varying workpieces, for example, the clamping module can be easily changed without modification of the machine tool. 


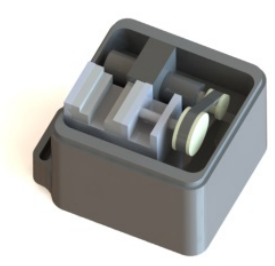

Clamping Module
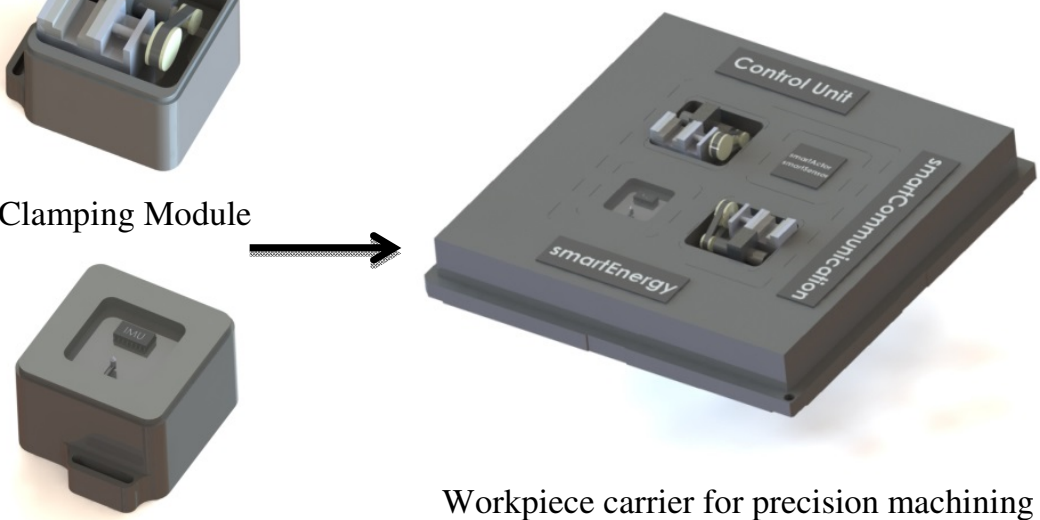

Workpiece carrier for precision machining

IMU + Temperature module

Fig. 3. Components of workpiece carrier for precision machining

\section{Discussion}

Compared to other work in the field of workpiece carriers in this paper a concept for a flexible and modular system, adapted to the requirements in micro assembly and related micro production processes requiring high precision is presented.

Beyond conventional passive workpiece carriers the modular electronic platform enables the integration of process and monitoring functionality. Its own energy supply allows, combined with the wireless data transfer capability, the continuous track and trace in production of quality and safety related devices. Due to the energy supply also the acquisition of measurement data or the control of actors is possible, even outside machines during transport or storage.

The modular design enables the precise adaption to the product and processes without dissipation of resources by overdesigned or unutilized features of the workpiece carrier. The standardization of the modules and their interfaces simplifies the adaption and makes reusability of modules possible.

The next tasks will be the implementation of the single elements of the modular electronic platform and the modular mechanical design as well as the merging to workpiece carrier systems. These systems will be technically and economically validated in several industrial use cases in micro assembly, ultra-precision machining and precision measurement.

Acknowledgements. This paper has emerged from the project »smartWT«, which has been sponsored by the German Federal Ministry of Education and Research (BMBF) in the funding program "Spitzencluster MicroTEC Südwest" and is administered by the project sponsor VDI/VDE-IT in Berlin. 


\section{References}

[1] Westkämper, E. (ed.): Wandlungsfähige Produktionsunternehmen: Das Stuttgarter Unternehmensmodell, pp. 7-23. Springer, Heidelberg (2009)

[2] Schilp, J.: Adaptive Montagesysteme für hybride Mikrosysteme unter Einsatz von Telepräsenz, vol. 244. Herbert Utz Verlag (2012)

[3] ElMaraghy, H.A.: Changeable and reconfigurable manufacturing systems. Springer (2009)

[4] Pfeffer, M., Goth, C., Craiovan, D., Franke, J.: 3D-Assembly of Molded Interconnect Devices with standard SMD pick \& place machines using an active multi axis workpiece carrier. In: 2011 IEEE International Symposium on Assembly and Manufacturing (ISAM), May 25-27, pp. 1-6 (2011)

[5] Shea, K., Ertelt, C., Gmeiner, T., Ameri, F.: Design-to-fabrication automation for the cognitive machine shop. Advanced Engineering Informatics 24(3), 251-268 (2010)

[6] Brecher, C., Manoharan, D., Ladra, U., Köpken, H.-G.: Chatter suppression with an active workpiece holder. Prod. Eng. Res. Devel. 4(2-3), 239-245 (2010)

[7] Abele, E., Hanselka, H., Haase, F., Schlote, D., Schiffler, A.: Development and design of an active work piece holder driven by piezo actuators. Prod. Eng. Res. Devel. 2(4), 437-442 (2008)

[8] Adamietz, R., Iseringhausen, T., Schlenker, D.: VolProd - Survey among microsystem manufacturers in Germany. In: Fraunhofer IPA (2012) 